Supporting Information

\title{
Adapting the electron beam from SEM as a quantitative heating source for nanoscale thermal metrology
}

\author{
Pengyu Yuan ${ }^{\S, 1,2,3}$, Jason Y. Wu ${ }^{\S, 1}$, D. Frank Ogletree ${ }^{2}$, Jeffrey J. Urban ${ }^{2}$, Chris Dames ${ }^{1, *}$, \\ Yanbao $\mathrm{Ma}^{3, *}$
}

${ }^{1}$ Department of Mechanical Engineering, University of California, Berkeley, CA 94720, USA

2 The Molecular Foundry, Lawrence Berkeley National Laboratory, Berkeley, CA 94720, USA

${ }^{3}$ Department of Mechanical Engineering, University of California, Merced, CA 95343, USA

$\S$ P. Y and J. Y. W. contributed equally to this work.

Correspondence and requests for materials should be addressed to C.D. (email: cdames@,berkeley.edu) and Y. M. (email: yma5@ucmerced.edu) 


\section{CASINO Calculation details}

The CASINO software package calculates the electron trajectories within the specimen using Monte Carlo methods to model the various physical interactions between the electrons and the sample ${ }^{1}$. The program tracks the electron trajectories as dictated by the elastic scattering events (see example in Fig. S1), while the inelastic events between every consecutive pair of elastic scatterings are estimated by the mean energy loss model. The absorbed energy from the e-beam is from the inelastic events, which in CASINO are approximated as being uniformly distributed along the electron's path between consecutive elastic scattering events. A continuous slowing-down equation is used to model this phenomenon (electron energy loss per unit path length), which combined with an estimation of the stopping energy determines the lateral and vertical electron range, as well as the corresponding spatial distribution of the inelastic energy transfer. In CASINO, several models can be selected for different interaction conditions. We complete the calculation of each electron trajectory by the following steps. First, the initial voltage (various, from $2 \mathrm{kV}$ to 20 $\mathrm{kV}$ ), direction (normally incident on the sample surface), and diameter ( $3 \mathrm{~nm}$ ) of the e-beam are specified. Note that we varied the diameter of the e-beam and found that the absorbed energy is insensitive to the diameter varying from $1 \mathrm{~nm}$ to $10 \mathrm{~nm}$. For each electron, the distance between consecutive elastic collisions is obtained based on the total elastic scattering cross-section, in which a random number is used to distribute the distance following a probability distribution. The elastic scattering angle is then determined from another random number and using the differential cross-section from standardized tabulated values. These steps are then repeated until an electron leaves the sample or is trapped inside the sample based on the stopping energy. Here we use a piecewise model of the energy loss function, which combines one model (ref. [2]) for low e-beam energies $(<10 \mathrm{keV})$ and the Bethe (1930) equation for high e-beam energies $(10-30 \mathrm{keV})$, which 
together provide a well-accepted and simple analytical expression for the stopping power ${ }^{3}$. The cross-section representing the distance between elastic collisions is computed using the tabulated Mott elastic scattering cross-sections of Czyzewski et al ${ }^{4}$. To obtain a sufficiently small statistical error, a total of 50,000 electron trajectories are calculated for each condition. Finally, the cumulative statistics of all 50,000 electron trajectories are used to determine the distribution of absorbed energy throughout the sample and calculate the total absorption fraction, $\alpha$.

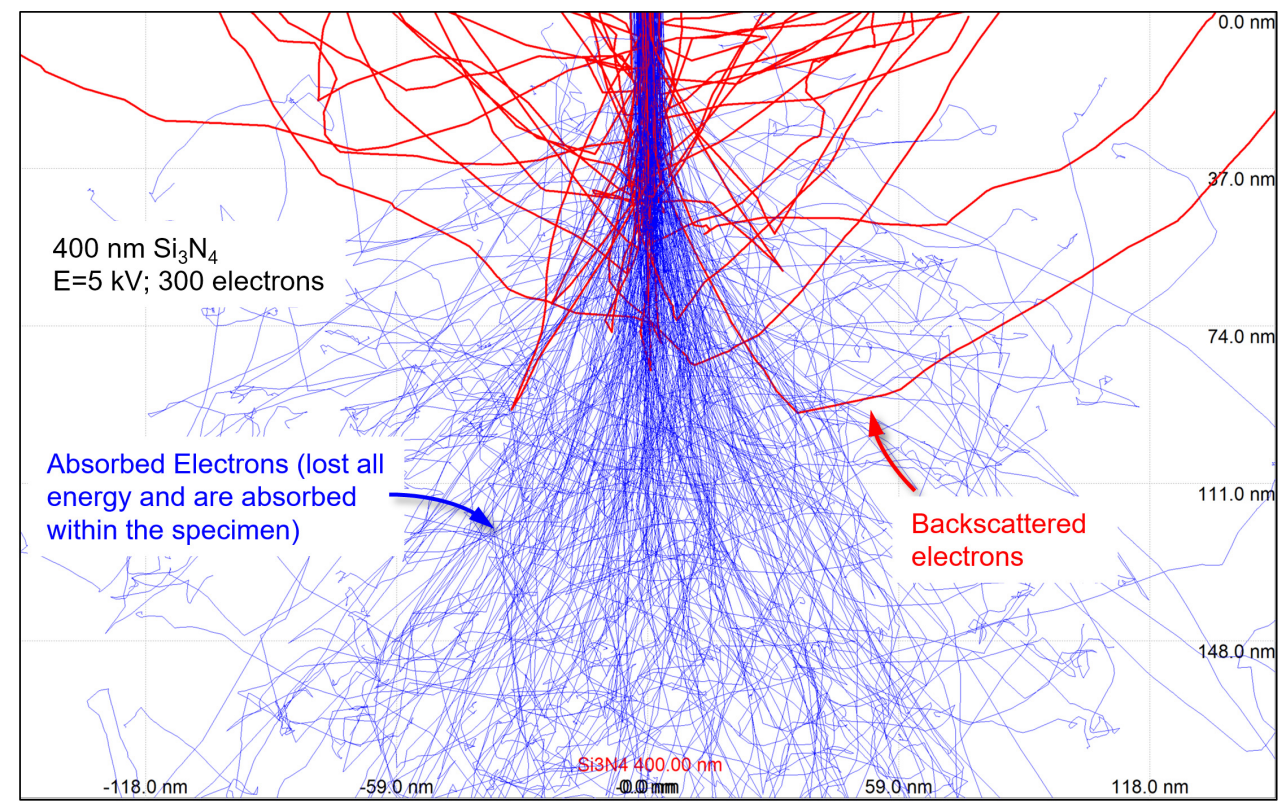

Figure S1. Example of CASINO Monte Carlo electron trajectories for $400 \mathrm{~nm}$ thick $\mathrm{Si}_{3} \mathrm{~N}_{4}$ thin film. Here only 300 electron trajectories are shown to make it easier to differentiate the absorbed electrons (blue) and the backscattered electrons (red). Only the top $185 \mathrm{~nm}$ of the sample is shown.

\section{Device design and fabrication}

The microfabricated low-stress $\operatorname{SiN} x$ calorimeter devices employed in this work feature a $21 \mu \mathrm{m}$ (width) $\times 20 \mu \mathrm{m}$ (height) suspended island region that is thermally isolated from the substrate by four $300 \mu \mathrm{m}$ long suspension beams each with a width of $1 \mu \mathrm{m}$. The SiN $x$ thickness varies from 
200 to $500 \mathrm{~nm}$. On top of the $\mathrm{SiN} x$ thin film is a 4-probe PRT consisting of a long serpentine line (shown in light green in Fig. 1c) with a resistance of $\sim 3.7 \mathrm{k} \Omega$ between the voltage measuring probes. The serpentine lines are $\sim 500 \mathrm{~nm}$ wide and $\sim 30 \mathrm{~nm}$ thick platinum. As shown in Fig. $2 \mathrm{~b}$, one Pt contact pad is integrated into each of the four suspension beams, thereby enabling fourpoint resistance thermometry of the serpentine PRT.

As shown in Fig. S2, for both the calorimeter and the 1D ribbon devices, the fabrication process started with low-stress low-pressure chemical vapor deposition (LPCVD) of silicon nitride ( $\mathrm{SiN} x$ ) on a 6-inch diameter, $500 \mu \mathrm{m}$ thick, p-type (100) Si wafer. The nominal thicknesses of the SiNx thin films are confirmed by a profilometer to be within $1 \%$ variance, thermal conductivity uncertainty calculations. Then $5 \mathrm{~nm}$ of $\mathrm{Cr}$ and $30 \mathrm{~nm}$ of Pt were deposited by electron beam evaporation. Then a $200 \mathrm{~nm} \mathrm{SiO} 2$ hard mask was deposited on the metal by plasma-enhanced chemical vapor deposition (PE-CVD). For patterning, firstly, standard photolithography was performed with an AMSL DUV (deep ultraviolet) stepper Model 5500/300, then the pattern was transferred to the $\mathrm{SiO}_{2}$ by fluorine-based RIE (reactive ion etch) dry etching. The $\mathrm{Cr} / \mathrm{Pt}$ was then patterned by ion milling using the hard mask. Secondly, the low-stress nitride was patterned by the stepper and RIE etching. This also defined the etch window for the $\mathrm{Si}$ etch. The $\mathrm{SiO}_{2}$ hard mask was removed by a buffered oxide etch (BOE) solution before releasing the nitride membrane using a $40 \% \mathrm{KOH}$ solution in DI water.

Note that for the $\mathrm{Si}$ etch to release the suspended $\mathrm{SiN} x$ structures, considering the orientationselective etch of the $\mathrm{Si}$ wafer in $\mathrm{KOH}$ solution, the etch depth into the wafer $(z)$ will be directly controlled by the lateral size $(x y)$ of the etch window. In the completed device, during the e-beam interaction with the SiN $x$ thin film, any transmitted electrons could potentially be reflected by the deep bottom of the Si trench. As depicted in the last panel of Fig. S2, some of these reflected 
electrons have the potential to return to the suspended $\mathrm{SiN} x$ structure, which would alter the charge and energy balances as compared to the idealized single-electron-pass configuration of the CASINO simulations. We minimize such potential back reflections by using a large trench depth, which minimizes the "electron view factor" between the bottom of the trench and the suspended SiN $x$ beam. Accordingly, the narrower of the two widths of the etch window (i.e., the vertical $(y)$ direction in Fig. 2b) is defined as $400 \mu \mathrm{m}$, which gives the etch depth of around $400 \mu \mathrm{m} / \sqrt{2}=283$ $\mu \mathrm{m}$. We prefer not to etch completely through the wafer in order to maintain the mechanical robustness of the device to increase the yield rate during the subsequent device separation and sample preparation process.

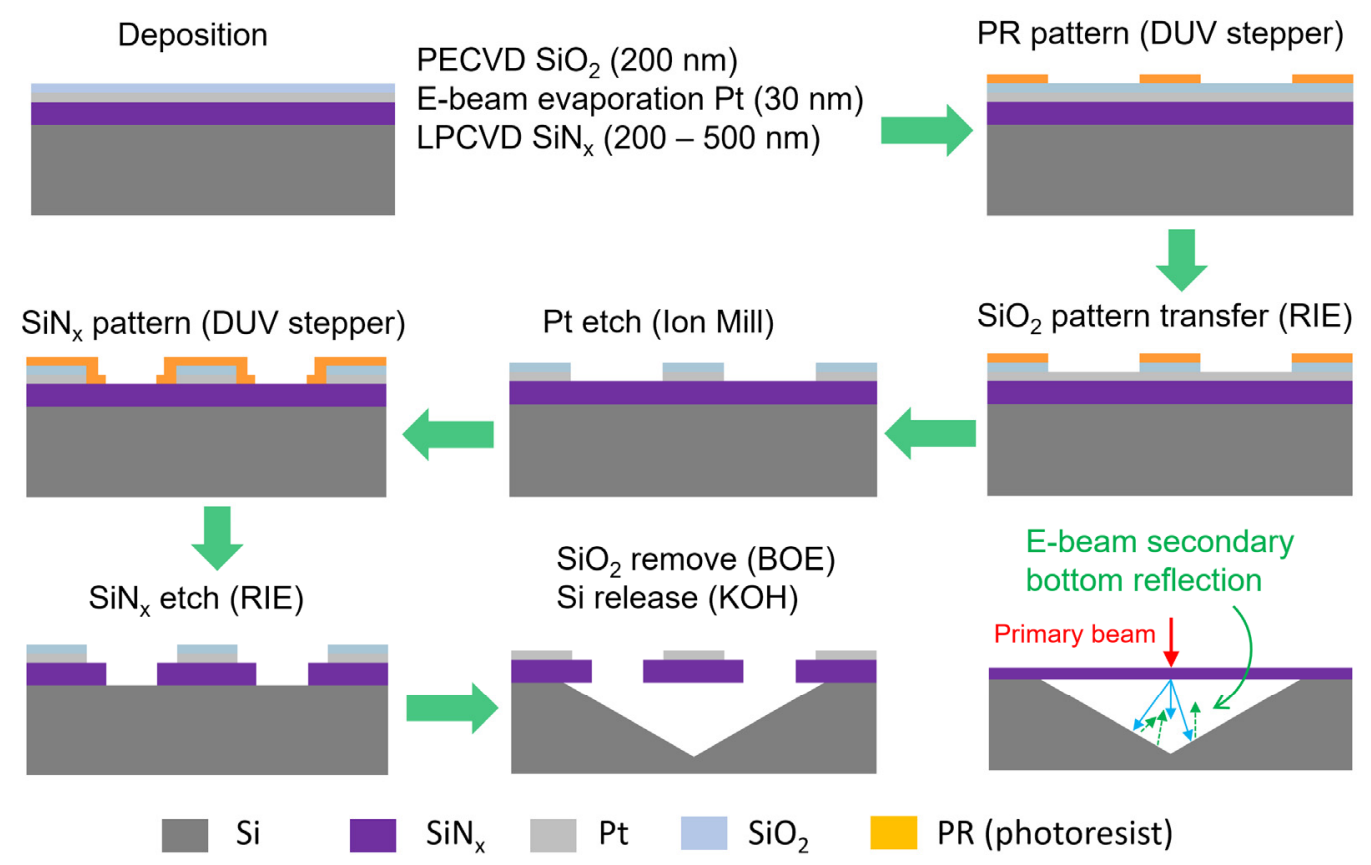

Figure S2. The device fabrication process, starting from layer deposition, then pattern transfer to Pt and $\operatorname{SiN} x$, which also defines the Si etch window. The final step is releasing the $\operatorname{SiN} x$ thin-film structures. The bottom right figure depicts a possible electron beam reflection at the deep bottom of the trench. 


\section{Device thermal Characterization}

To measure small temperature changes with the PRT it is first necessary to calibrate its temperature coefficient of resistance (TCR), denoted with the symbol $\eta$, and defined as $\eta(T)=(d R / d T) / R(T)$. Then, the change in the temperature of the resistor $(\Delta T)$ can be directly obtained from $\Delta T=\Delta V_{\mathrm{s}} /\left[I_{\mathrm{s}} \times R_{4 \mathrm{p}}(T) \times \eta(T)\right]$, where $\Delta V_{\mathrm{s}}$ is the voltage change, $I_{\mathrm{s}}$ is the input sensing current, and $R_{4 \mathrm{p}}$ is the 4-probe electrical resistance of the PRT serpentine. We measured this $\Delta R_{4 \mathrm{p}}$ using an SR830 lock-in amplifier with a $200-500 \mathrm{nA}, 878 \mathrm{~Hz}$ sinusoidal excitation current. This sensing current is small enough to cause negligible self-heating $(<<1 \mathrm{mK}$ temperature rise). Calibration details can be found in the next section. Also, to use the calorimeter we need to determine the total thermal conductance $(G)$ of the four suspension beams including the Pt film. As detailed in the Supporting Information Section 4\&5, following Ref. 5 this was accomplished in a separate calibration step by passing a DC current through the PRT and measuring the corresponding temperature rise of the central island.

\section{Temperature coefficient of resistance (TCR)}

For both calorimeter and 1D ribbon devices, the temperature coefficient of resistance (TCR), $\eta$, of the serpentine heater line was determined by measuring the line's electrical resistance $R_{4 \mathrm{p}}(T)$, at different temperatures $T$. This calibration was performed separately for every device. For each, the 4-probe resistance was measured by passing a small AC sensing current of amplitude $I_{\mathrm{AC}}=300$ nA (with frequency $\mathrm{f}=878 \mathrm{~Hz}$ ) through the device, resulting in negligible self-heating. As shown in Fig. S3, the corresponding $\mathrm{AC}$ voltage across the PRT $\left(V_{\mathrm{AC}}\right)$ was measured with a lock-in amplifier in a 4-probe configuration, and the resistance of the PRT is calculated as $R_{4 \mathrm{p}}=V_{\mathrm{AC}} / I_{\mathrm{AC}}$. 
In this work, we used a lock-in temperature measurement scheme with a modulated sensing current which can give a noise equivalent temperature (NET) resolution of around $25 \mathrm{mK}$ (at 300K). This is sensitive enough to characterize the heating from the electron beam in the present work, and the circuit is fairly simple. Therefore we did not need to consider other, more highly sensitive thermometry techniques like a Wheatstone bridge scheme with a NET as small as $\sim 0.05 \mathrm{mK}$ (at $300 \mathrm{~K})^{6}$, though for resistance thermometers with resistance less than $1 \Omega$ this might more practical than the current approach. Another option would be a DC reversal method with a NET of $\sim 2 \mathrm{mK}$ which has been recently demonstrated, though that requires more instrumentation? ${ }^{7}$

A high-vacuum cryostat was used for all thermal characterization and was evacuated to better than $5 \times 10^{-6}$ Torr using a turbomolecular pump. A commercial PID temperature controller (Lakeshore 331) was used to stabilize the temperature of the cold head of the cryostat, with a typical stability of $\pm 0.02 \mathrm{~K}$. Because these experiments make only small excursions from the ambient reference temperature $T_{0}$, the resistance can be linearized as

$$
R_{4 \mathrm{P}}(T)=R_{4 \mathrm{P}}\left(T_{0}\right) \times(1+\eta \Delta T),
$$

where $\Delta T=T-T_{0}$. Therefore

$$
\frac{R_{4 \mathrm{P}}(T)-R_{4 \mathrm{P}}\left(T_{0}\right)}{R_{4 \mathrm{P}}\left(T_{0}\right)}=\frac{\Delta R_{4 \mathrm{P}}(T)}{R_{4 \mathrm{P}}\left(T_{0}\right)}=\eta \Delta T
$$

From the calibration experiments $\eta$ is determined for every device from its slope of $\Delta R_{4 \mathrm{p}}$ vs. $\Delta T$ (e.g., as in Fig. S3d for a representative serpentine). The TCR calibration results for all devices can be found in Table S1.

Table S1. Summary of thermal characterization results of all calorimeter and 1D ribbon devices at room temperature. Error bars correspond to the standard error of the linear regression. 


\begin{tabular}{ccccc}
\hline & & & & Calorimeter \\
SiNx & Calorimeter TCR & 1D ribbon PRT 1 & 1D ribbon $\mathrm{PRT}_{2}$ & beam thermal \\
Thickness & $\left(\times 10^{-3} \mathrm{~K}^{-1}\right)$ & TCR $\left(\times 10^{-3} \mathrm{~K}^{-1}\right)$ & TCR $\left(\times 10^{-3} \mathrm{~K}^{-1}\right)$ & $\left(\times 10^{-8} \mathrm{WK}^{-1}\right)$ \\
\hline $200 \mathrm{~nm}$ & $1.980 \pm 0.012$ & $2.080 \pm 0.052$ & $1.987 \pm 0.056$ & $3.253 \pm 0.006$ \\
$300 \mathrm{~nm}$ & $1.590 \pm 0.010$ & $2.183 \pm 0.051$ & $2.128 \pm 0.016$ & $3.453 \pm 0.005$ \\
$400 \mathrm{~nm}$ & $0.986 \pm 0.002$ & $2.906 \pm 0.036$ & $2.795 \pm 0.020$ & $4.060 \pm 0.007$ \\
$500 \mathrm{~nm}$ & $0.860 \pm 0.003$ & $3.263 \pm 0.055$ & $3.252 \pm 0.067$ & $3.592 \pm 0.008$ \\
\hline
\end{tabular}
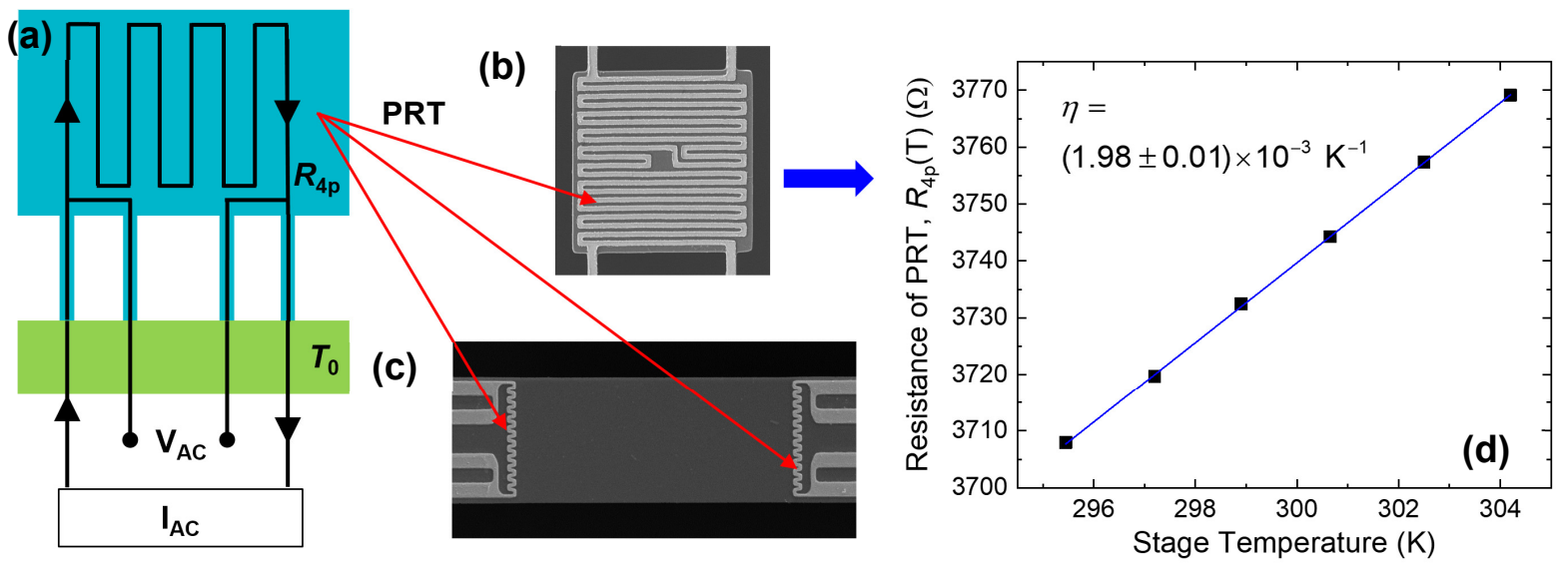

Figure S3. TCR measurement. (a) Schematic of the TCR measurement. (b) Micrograph showing 4-point connections for a PRT on a calorimeter device. (c) Similarly, two PRTs on a 1D ribbon device. (d) Measured resistance of the PRT on the $200 \mathrm{~nm}$ thick calorimeter at different cryostat stage temperatures.

\section{Thermal conductance of supporting beams of calorimeter devices}


Figure S4 shows a schematic of the experimental setup for measuring the total thermal conductance $(G)$ of the suspending beams used in the calorimeter devices. Each device was characterized individually in the same high-vacuum cryostat as used for the TCR measurements described above. A DC current $I_{\mathrm{DC}}$ flows through the PRT, generating a Joule heating $Q_{h}=I_{\mathrm{DC}}^{2} \times R_{4 \mathrm{p}}$ in the island's serpentine line with its resistance $R_{4 \mathrm{p}}$. As indicated in Fig. S4a, a much smaller AC current was superposed on $I_{\mathrm{DC}}$ to enable resistance thermometry of $R_{4 \mathrm{p}}$ by lock-in detection of $V_{\mathrm{AC}}$, which contributes negligible additional Joule heating because $I_{\mathrm{AC}}<<I_{\mathrm{DC}}$. We also considered the finite resistance of each Pt current lead, $R_{L}$, which in these devices is typically about half of $R_{4 \mathrm{p}}$ and was measured separately by a two-probe method. A combined Joule heat of $2 Q_{L}=2 I_{\mathrm{DC}}^{2} \times R_{\mathrm{L}}$ is dissipated in the two Pt leads that supply the DC current to the central serpentine PRT. The temperature of the heating island remains spatially uniform at $T_{h}$, because the internal thermal resistance of the island is much smaller than the combined thermal resistance of the four slender beams, $G^{-1}$. The Joule heat of $Q_{L}$ was distributed uniformly along each of the two Pt leads supplying the heating IDC current, yielding a parabolic temperature distribution along these two beams, while a linear temperature profile will occur in the remaining two beams without Joule heating. Following ref. [5], the heat conduction to the environment from both Joule-heated beams can be derived as $Q_{1}$,

$$
Q_{1}=2 \times\left(\frac{G}{4} \times \Delta T_{\mathrm{h}}+\frac{Q_{L}}{2}\right)
$$

where $G / 4$ is the thermal conductance of a single beam, while that from the remaining two beams is 


$$
Q_{2}=2 \times \frac{G}{4} \times \Delta T_{\mathrm{h}} .
$$

Considering energy conservation for the thin film as $Q_{1}+Q_{2}=Q_{h}+2 Q_{L}$, we then can obtain

$$
G=\frac{Q_{h}+Q_{L}}{\Delta T_{h}}=\frac{I_{D C}^{2}\left(R_{h}+R_{L}\right)}{\Delta T_{h}} .
$$

$Q_{h}$ and $Q_{L}$ can be calculated readily from the measured DC current and the voltage drops across the PRT and the PRT leads. $\Delta T_{h}$ is evaluated from the AC measurements of the resistance of the PRT and knowledge of its TCR. As shown in Fig. 2b, $G$ at $300 \mathrm{~K}$ is about $3.25 \times 10^{-8} \mathrm{WK}^{-1}$, in good agreement with the value of $2.73 \times 10^{-8} \mathrm{WK}^{-1}$ which is calculated from the geometry of the four beams as well as the room temperature thermal conductivity values of $\mathrm{SiN} x$ and Pt films, i.e., $k \mathrm{SiN}_{x}=3.61 \mathrm{Wm}^{-1} \mathrm{~K}^{-1}$ from this Joule heating measurement, and $k \mathrm{Pt}_{\mathrm{Pt}}=$ 46.3 $\mathrm{Wm}^{-1} \mathrm{~K}^{-1}$ is estimated by Wiedemann-Franz law. The measured $G$ values for all calorimeters are summarized in Table S1.
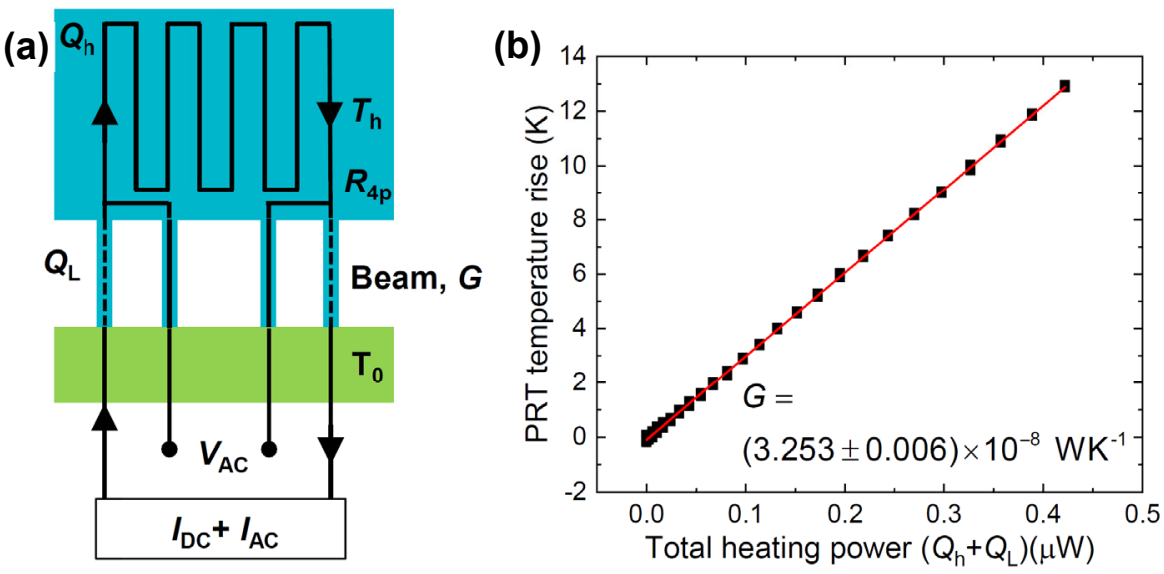
Figure S4. Calorimeter beam thermal conductance measurement details. (a) Measurement schematic. (b) The beam thermal conductance $G$ fitting result for the $200 \mathrm{~nm}$ thick device around room temperature. $G$ means the total thermal conductance of the four beams in parallel.

\section{Maximum temperature rise induced by e-beam heating}

Referring to Fig. 2c of the main text, here we present an estimate of the highly localized temperature rise in the immediate vicinity (10s of $\mathrm{nm}$ ) of the e-beam heating spot, $\Delta T_{\text {local. }}$. This is different than the overall temperature difference between the calorimeter test platform and surrounding wafer which is denoted $\Delta T=T$ - $T_{0}$ in the main text and which we measure directly. We approximate this heat conduction problem as $2 \mathrm{D}$ radial heat conduction in the plane of a thin film of thickness $t$, with a cylindrical heat source of radius $r_{1}$ representing the e-beam, and a heat sink located a distance $r_{2}$ away representing the average temperature of the test platform. Recognizing that the peak heating power in our experiments happens when the e-beam fully penetrates the sample, we approximate the heating volume as a cylindrical heat source of radius $r_{1}$ and length $t$, that is, the film thickness. The solution to this heat conduction problem is widely available in undergraduate textbooks on heat transfer ${ }^{8}$, and also is provided in a standard SEM textbook [Eq. 3.201 of Ref. 9]. The result is:

$$
\Delta T_{\text {local }}=\frac{Q}{2 \pi k t} \times \ln \left(\frac{r_{2}}{r_{1}}\right) .
$$

Here $Q=1 \mu \mathrm{W}$ is a typical e-beam heating power, $k=2 \mathrm{Wm}^{-1} \mathrm{~K}^{-1}$ is the thermal conductivity of the SiNx film, $t=200 \mathrm{~nm}$ is a typical film thickness, $r_{1}=1 \mathrm{~nm}$ is the e-beam radius, and we take $r_{2}=5 \mu \mathrm{m}$ representing the average distance between the e-beam interaction spot and the 
metal PRT lines in Fig. $2 \mathrm{c}$ of the main text. Plugging in numbers, we have $\Delta T_{\text {local }} \approx 7 \mathrm{~K}$. In other words, the true temperature immediately under the $\sim 1 \mathrm{~nm}$ e-beam spot is approximately $7 \mathrm{~K}$ higher than the measured temperature from the PRT in Fig. 2c, and this hot spot decays to only a $3 \mathrm{~K}$ temperature rise at a distance $100 \mathrm{~nm}$ away from the center of the calorimeter platform. This is a small value in comparison to typical MEEHV conditions, in which the measured temperature rise of the calorimeter, between the PRT and the surrounding silicon wafer, is typically $60 \mathrm{~K}$.

\section{Joule heating method to determine the thermal conductivity of SiNx thin film}

For the Joule heating method depicted in Fig. 4 we apply a DC current to generate localized Joule heating at $\mathrm{PRT}_{1}$, similar to a localized e-beam line heater. However, as shown in Fig. S5a (top), this causes additional Joule heating in the two current leads, which we take into account using measurements with two different connections. In Case A of Fig. S5a, the current is applied between contact pad 1 and contact pad 4, while in Case B, the current was applied between contact pads 1 and 2, thereby bypassing the PRT. Then, these measurements are analyzed using a superposition argument to deduce the corresponding Case C, as discussed next.

(a) Superposition argument

From the principle of linear superposition, we recognize that Case A is very nearly equivalent to Case B plus Case C, where Case C corresponds to a localized line heat source only at the PRT which would be easiest to analyze. This superposition argument is not exact because in Case A leads 1 and 4 experience Joule heating, while in Case B it is leads 1 and 2. However, because the heat transfer is predominantly along the $x$-direction, and leads 2 and 4 differ only 
in their y coordinate, this distinction will have very little impact on the heat conduction problem. Another imperfection in the superposition is the Joule heating in Case B in the small metal connection between leads 1 and 2 (purple area in Fig. S5b, $2 \mu \mathrm{m} \times 0.75 \mu \mathrm{m}$ ), which is not present in Case $\mathrm{A}$. Overall, the equivalence $\mathrm{C} \approx \mathrm{A}-\mathrm{B}$ holds true to a very good approximation, as confirmed by 3D numerical simulations in Supporting Information section 7 and Fig. S6 below. Therefore, for the analysis of the Joule heating approach for extracting $k$ of the $\operatorname{SiN} x$ film, we measure $T_{1}$ and $T_{2}$ as functions of $I_{\mathrm{DC}}$ for Cases $\mathrm{A}$ and $\mathrm{B}$, and then determine the equivalent $T_{1 \mathrm{C}}$ and $T_{2 \mathrm{C}}$ by subtraction, for example $T_{1 \mathrm{C}}\left(I_{\mathrm{DC}}\right)=T_{1 \mathrm{~A}}\left(I_{\mathrm{DC}}\right)-T_{1 \mathrm{~B}}\left(I_{\mathrm{DC}}\right)$. Note that for the purposes of Fig. S7(a), IDC has been expressed as a corresponding $Q_{\mathrm{PRT} 1}$, this is defined as $I_{D C}^{2} R_{4 p}$, even for Case $\mathrm{B}$ with no heat actually dissipated in $\mathrm{PRT}_{1}$. This transformation to Case C simplifies the heat conduction analysis leading to Eqs. (11) - (15). Also, considering the obvious left-right symmetry of the 1D ribbon design, we repeat these $k$ measurements using mirrored connections, i.e. Joule heating $\mathrm{PRT}_{2}$ rather than $\mathrm{PRT}_{1}$. For a given device, the measured $k$ values are expected to be the same for measurements using $\mathrm{PRT}_{1}$ and $\mathrm{PRT}_{2}$, and the results in Table 2 confirm that they differ by no more than $\pm 4 \%$.

(b) Metal line leads resistance effect

We analyze the differences between these two Cases By considering the various metal line segments making up the leads, depicted in Fig. S5b.

For a single platinum line segment of length $L$, by Pouillet's law, its electrical resistance is $R=\rho \times L / A$, where $\rho$ is the electrical resistivity and $A=W t$ is the cross-sectional area, where $W$ is the linewidth and $t=30 \mathrm{~nm}$ is the thickness. For convenience, we introduce $\varphi=(\rho / 3 \mathrm{t})$ which is simply $1 / 3$ times the sheet resistance, thus $R=(3 L / W) \varphi$. This factor of 3 is chosen 
to yield convenient integer values later. Thus, for example, the green section in Fig. S5b has $L / W=20$ and so $R=60 \varphi$. Similar calculations give the other $\varphi$ values listed in Fig. S5b.

Using $R_{\mathrm{i}-\mathrm{j}}$ to denote the two-probe electrical resistance between pads $\mathrm{i}$ and $\mathrm{j}$, from Fig. $3 \mathrm{c}$ and using the $\varphi$ values enumerated in Fig. S5b we find $R_{1-4}=920 \varphi+R_{4 \mathrm{p}}$. Similarly, from Fig. S5d we have $R_{1-2}=889 \varphi$. Here, we can directly measure $R_{4 \mathrm{p}}$ by a four probe configuration, and $R_{1-2}$ and $R_{1-4}$ by a two probe configuration. However, the Pt sections indicated in green and yellow in Fig. S5b are substrate-supported with excellent heat sinking, and therefore do not contribute to the actual Joule heating. Therefore define $R_{\mathrm{B}}$ as the unsupported portion of $R_{1-2}$, and thus $R_{\mathrm{B}}=809 \varphi$. These geometric calculations allow us to eliminate $\varphi$ to evaluate $R_{\mathrm{B}}$ directly from the measured $R_{1-2}$, using $R_{\mathrm{B}}=(809 / 889) R_{1-2}$. Similarly, define $R_{\mathrm{A}}$ as the unsupported portion of $R_{1-4}$, and thus $R_{\mathrm{A}}=800 \varphi+R_{4 \mathrm{p}}$, whereas $R_{1-4}=920 \varphi+R_{4 \mathrm{p}}$; so eliminating $\varphi$ gives $R_{\mathrm{A}}=(20 / 23)\left(R_{1-4}-R_{4 \mathrm{P}}\right)$. Using the real electrical resistance for both Case $\mathrm{A}$ and Case $\mathrm{B}$, we can evaluate the Joule heating energy correspondingly. By subtracting the temperature rise of two PRTs and the Joule heating energy of Case B from Case A, we can have the Case C which can be directly used to determine the thermal conductivity of $\mathrm{SiN} x$ thin film.

(c) Thermal conductivity of $\mathrm{SiN} x$ ribbon from Joule heating method

In Joule heating method, we can eliminate $R_{\mathrm{L}}$ from Eqs 11 and 13 to have

$$
\frac{Q_{\text {PRT1_1 }}}{Q_{\text {PRT1_2 }}}=\frac{\Delta T_{1 \mathrm{C}}}{\Delta T_{2 \mathrm{C}}} .
$$

From Eqs 12 and 14, we eliminate $R_{\mathrm{L}}$ to express $R_{\mathrm{S}}$ as

$$
R_{\mathrm{S}}=\frac{\left(\Delta T_{1 \mathrm{C}}-\Delta T_{2 \mathrm{C}}\right) \times\left(\Delta T_{1 \mathrm{C}}+\Delta T_{2 \mathrm{C}}\right)}{Q_{\mathrm{PRT} 1} \times \Delta T_{2 \mathrm{C}}} .
$$


Differentiating Eq S7 and Eq S8 with respect to $Q_{\mathrm{PRT} 1}$, we can rewrite Eq S8 as

$$
R_{\mathrm{S}}=\left(\frac{\partial T_{1 \mathrm{C}} / \partial Q_{\mathrm{PRT} 1}}{\partial T_{2 \mathrm{C}} / \partial Q_{\mathrm{PRT} 1}}+1\right) \times\left(\frac{\partial T_{1 \mathrm{C}}}{\partial Q_{\mathrm{PRT} 1}}-\frac{\partial T_{2 \mathrm{C}}}{\partial Q_{\mathrm{PRT} 1}}\right) .
$$

Rearranging Eq S9 gives the $k$ expression as Eq 15.

Table S2. Complete in-plane thermal conductivity measurement results from e-beam and Joule heating methods. The $1^{\text {st }}$ Joule heating method is by using $\mathrm{PRT}_{1}$, and $2^{\text {nd }}$ is by using $\mathrm{PRT}_{2}$.

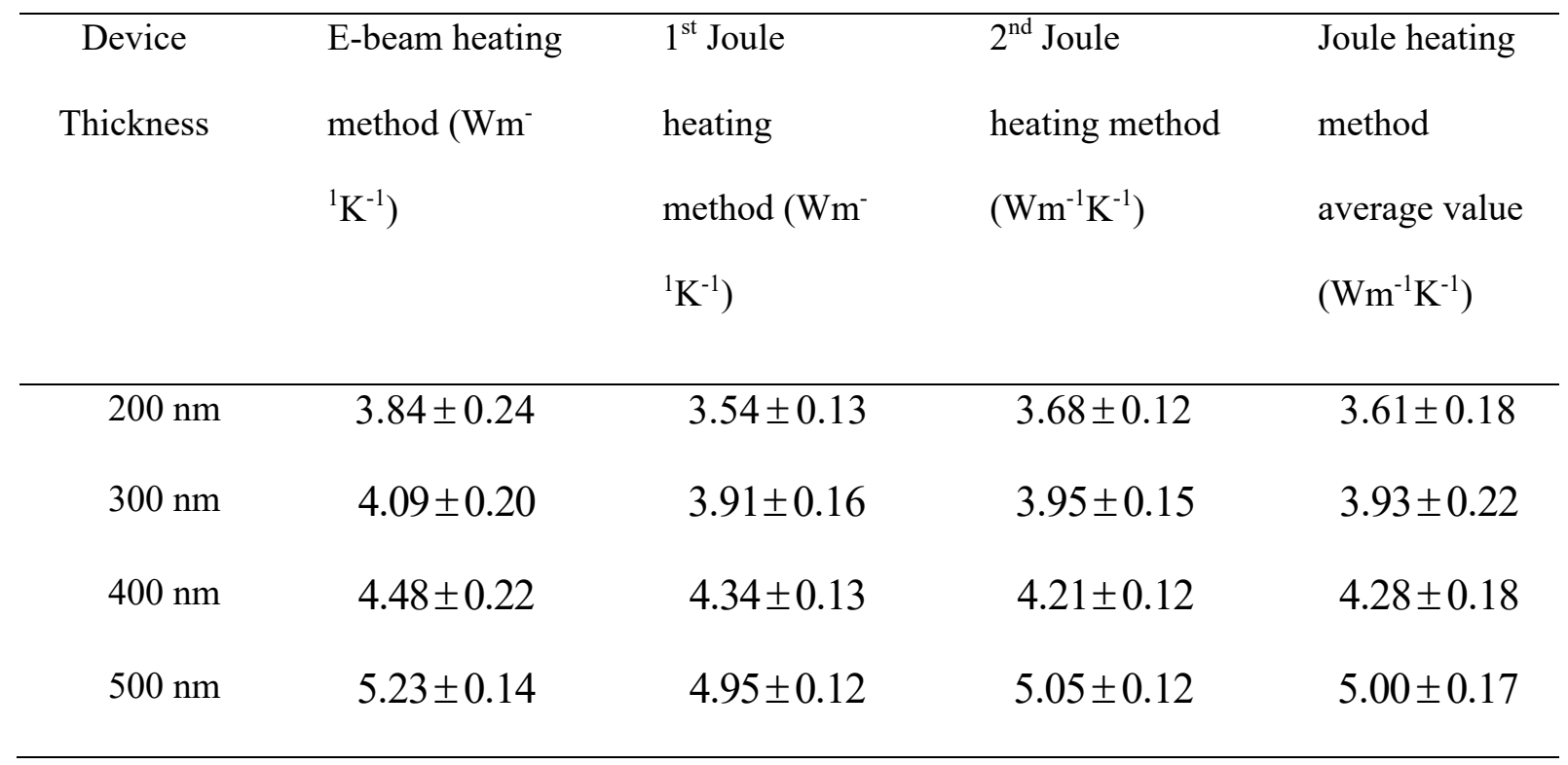



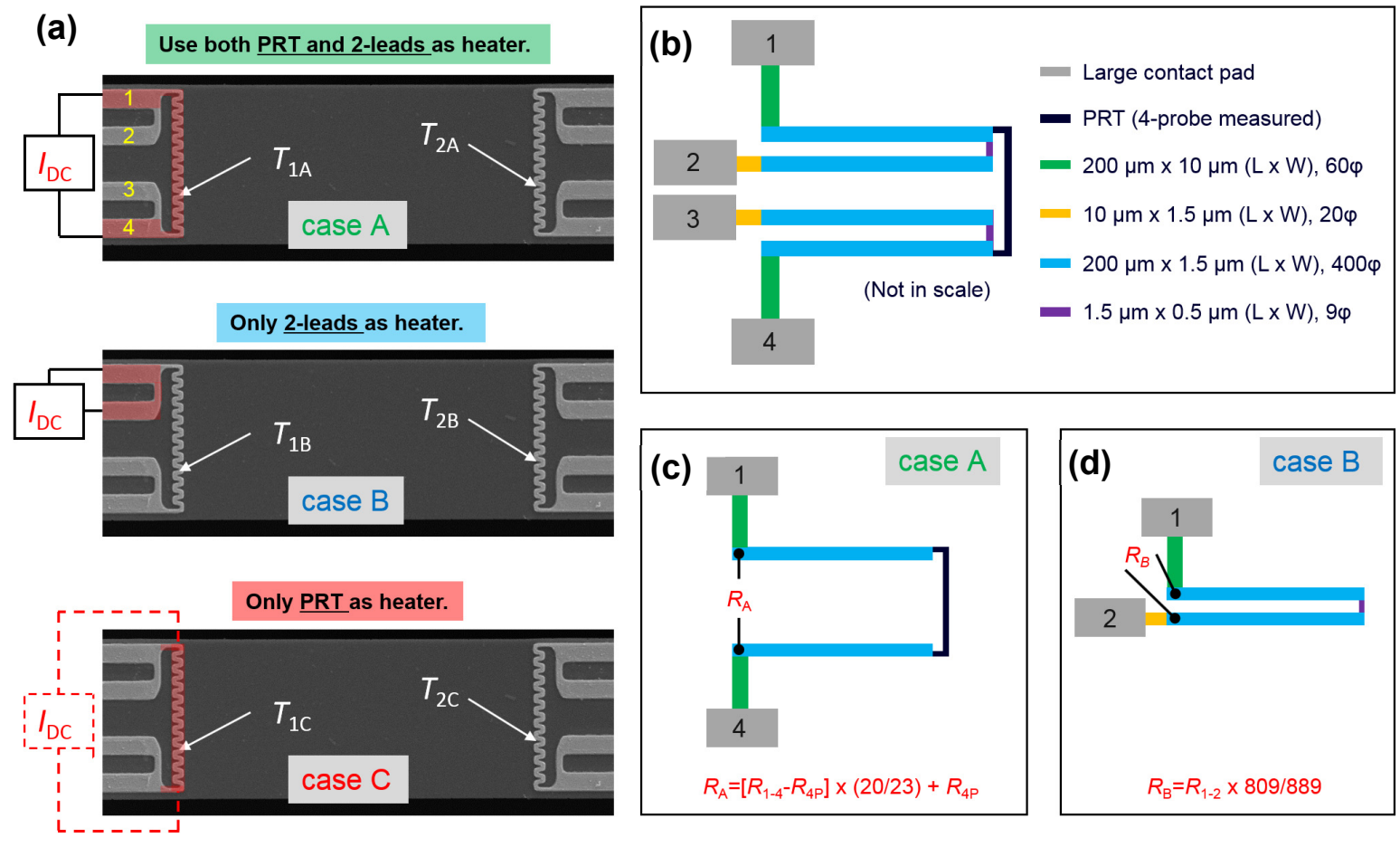

Figure S5. Variable connection scheme to realize the equivalent of localized Joule heating, in which the preferred situation, Case C, is approximated by the subtraction A - B. (a) Experimental connections for Cases A and B, and depiction of the ideal Case C. Red shading indicates regions of Joule heating. (b) Schematic of the electrical resistances of the various leads comprising the PRT. (c) Contributions to the resistance of Case A. (d) Contributions to the resistance of Case B.

\section{Numerical validation of the Joule heating method superposition}

As discussed in Supporting Information section 6, we approximate the temperature rise in Case $\mathrm{C}$ as the subtraction of that of Case B from Case A. Because the superposition is not exact, we check this approximation using 3D COMSOL simulations of the exact temperature distributions of $200 \mathrm{~nm} 1 \mathrm{D}$ ribbon sample in Cases A, B, and C, as shown in Fig. S5. As shown 
in Fig. S5 (c) and (d), for the various different colored zones (e.g. lead, PRT, etc.), we generate Joule heating in each zone in proportion to its electrical resistance. In Case A, for the total blue areas, we input heat flow as $P_{0}=1 \mu \mathrm{W}$, chosen as a convenient reference condition. Accordingly, we proportionally heat the PRT area as $\left[R_{4 \mathrm{P}} /\left(R_{\mathrm{A}}-R_{4 \mathrm{P}}\right)\right] \times P_{0}$. Similarly, for Case $\mathrm{B}$, we input $P_{0}$ into the two blue areas, and the small connection area (purple area) as $(9 / 809) \times P_{0}$. Then we check the superposition by simply summing the temperature profiles of Cases B and C. The resulting green curve is a little bit below the temperature profile from Case A with less than $1 \%$ difference. Therefore, we conclude that the superposition approximation is suitable here.

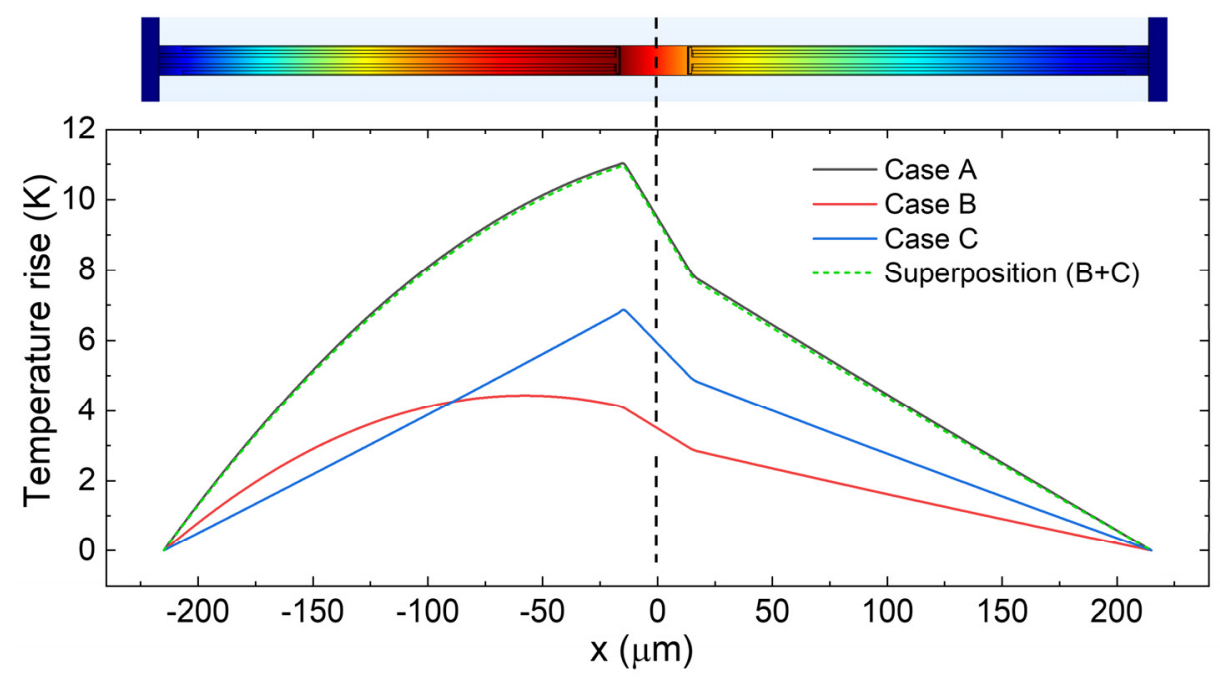

Figure S6. The COMSOL simulation results for Joule heating method to validate the superposition assumption. The upper image shows the COMSOL-generated temperature profile from Case A. The plotted temperature in the lower panel is. taken from the sample surface. 


\section{Supporting Information References}

1. Hovington, P., Drouin, D., and Gauvin, R., "CASINO: A new Monte Carlo code in C language for electron beam interaction-Part I: Description of the program," Scanning 19, 1-14 (1997).

2. Joy, D. C., Monte Carlo modeling for electron microscopy and microanalysis. Oxford University Press: 1995; Vol. 9.

3. Lowney, J. R., "MONSEL-II Monte Carlo Simulation of SEM Signals for Linewidth Metrology," $J$. Microbeam Analysis 4, (1995).

4. Czyżewski, Z., MacCallum, D. O. N., Romig, A., and Joy, D. C., "Calculations of Mott scattering cross section," Journal of Applied Physics 68, 3066-3072 (1990).

5. Shi, L., Li, D., Yu, C., Jang, W., Kim, D., Yao, Z., Kim, P., and Majumdar, A., "Measuring thermal and thermoelectric properties of one-dimensional nanostructures using a microfabricated device," Journal of heat transfer 125, 881-888 (2003).

6. Zheng, J., Wingert, M. C., Dechaumphai, E., and Chen, R., "Sub-picowatt/kelvin resistive thermometry for probing nanoscale thermal transport," Review of Scientific Instruments 84, 114901 (2013).

7. Wu, J. Y., Wu, W., and Pettes, M. T., "Ultra-high resolution steady-state micro-thermometry using a bipolar direct current reversal technique," Review of Scientific Instruments 87, 094901 (2016).

8. Bergman, T. L., Incropera, F. P., DeWitt, D. P., and Lavine, A. S., Fundamentals of heat and mass transfer. John Wiley \& Sons: 2011.

9. Reimer, L., Electron scattering and diffusion. In Scanning Electron Microscopy, Springer: 1998; pp 57134. 